\title{
Penicillinase-producing Neisseria gonorrhoeae ophthalmia neonatorum in Singapore
}

\author{
T THIRUMOORTHY, V S RAJAN, AND C L GOH \\ From the Middle Road Hospital, Singapore, Republic of Singapore
}

SUMMARY The problem of ophthalmia neonatorum due to penicillinase-producing Neisseria gonorrhoeae (PPNG) has grown with the increasing prevalence of PPNG strains in Singapore. The epidemiological trends, clinical features, and treatment of ophthalmia neonatorum due to PPNG have been determined from the case histories of 11 babies with this condition treated between 1978 and 1980. All 11 cases were cured with a single intramuscular injection of kanamycin $0.5 \mathrm{~g}$ and $1 \%$ kanamycin eyedrops instilled for three days. In six of the 11 mothers, a non-PPNG strain was isolated.

\section{Introduction}

Gonococcal ophthalmia neonatorum is a preventable complication of gonorrhoea. Its low incidence is often used by epidemiologists as a measure of the effective control of gonorrhoea within a community. Since the advent and use of antibiotics early in the course of the disease, ophthalmia neonatorum is not now an important cause of blindness.

Although ophthalmia neonatorum is less commonly caused by Neisseria gonorrhoeae 1 in Western Europe the gonococcus is still the main cause of this condition in Singapore. In 1976 the first strains of PPNG were isolated in Singapore. ${ }^{2}$ Since then the incidence of PPNG has increased progressively so that $30 \%$ of all cases of gonorrhoea are presently caused by PPNG.

This paper reports our experience in Singapore of ophthalmia neonatorum due to PPNG between 1978 and 1980 and describes the epidemiological trends, clinical features, treatment, and the results of the microbiological investigation of parents of infected children.

\section{Patients and methods}

All case records of babies with ophthalmia neonatorum due to PPNG were reviewed. These babies had been admitted as inpatients to the Middle Road Hospital and were seen by one of us. There were 12 cases of ophthalmia neonatorum due to PPNG and 11 were available for detailed study.

Address for reprints: Dr V S Rajan, Middle Road Hospital, Middle Road, Singapore 0718, Republic of Singapore

Accepted for publication 26 April 1982

\section{DIAGNOSIS}

In all babies with conjunctivitis seen at the Middle Road Hospital swabs are taken from both eyes for Gram staining and microscopy and immediate culture on modified Thayer-Martin media. Babies in whom Gram-negative diplococci were seen on stained smears of exudate were diagnosed presumptively as having gonococcal ophthalmia neonatorum and were admitted as inpatients. If the mothers attend with their babies they are encouraged to be admitted with them. Using the iodometric method all cultures of $N$ gonorrhoeae are tested routinely for penicillinase activity. The result is known on the third, or at the latest, fourth day of admission.

Mothers were examined for gonococcal infection by microscopy of Gram-stained smears and by culture of material from urethra, cervix, and rectum. If gonococcal infection was not diagnosed at the initial attendance, these tests were repeated on two consecutive days. Fathers of babies with ophthalmia neonatorum were investigated at the clinic.

Culture specimens for Chlamydia trachomatis were not taken from the baby, the mother, or the father.

In babies who were being treated the amount of conjunctival discharge was recorded at least four times a day and smears and cultures were performed daily.

\section{TREATMENT}

The treatment of ophthalmia neonatorum consisted of parenteral injections of antibiotics and penicillin eyedrops instilled into each eye every five minutes for the first $\mathbf{3 0}$ minutes, every $\mathbf{3 0}$ minutes for three hours, and every two hours for $\mathbf{4 8}$ hours. 


\section{Results}

\section{EPIDEMIOLOGICAL TRENDS}

Since 1976, when the incidence of gonococcal ophthalmia in Singapore was 1.02 cases per 1000 live births, the incidence has fallen to 0.63 cases per 1000 live births in 1977. Since then there has been a gradual decrease to 0.56 cases per 1000 live births in 1980.

The first case of ophthalmia neonatorum caused by PPNG was reported in 1978 by Pang et al $^{3}$ from Singapore. The number of cases of ophthalmia neonatorum due to PPNG from 1976 to 1980 are shown in table I. In 1978 and 1979 two cases of ophthalmia neonatorum due to PPNG were reported and in 1980 there were eight cases. Of the 11 babies studied, four were male and seven were female; eight were Chinese, two Malay, and one Indian.

\section{CLINICAL FEATURES}

The incubation period varied from one to 11 days; the mode was three days. In all but one case the conjunctivitis was detected within six days of birth.

Nine of the 11 babies had a purulent discharge from both eyes; in the other two only the left eye was affected. Four babies had oedema and redness of both eyelids; three had minimal discharge and mild palpebral conjunctivitis; and four had moderate to mild inflammation.
In nine of the 11 babies the time between the onset of symptoms and the start of specific treatment for PPNG infection was six days or longer, since the first-line treatment of gonococcal ophthalmia neonatorum was penicillin eyedrops followed by intramuscular benzylpenicillin.

\section{TREATMENT}

On the basis of the finding of Gram-negative intracellular diplococci in conjunctival smears, nine babies were started initially on penicillin eyedrops; one baby was treated with chloramphenicol eyedrops and the other with kanamycin eyedrops. The initial antibiotic used and the clinical and bacteriological responses are shown in table II.

The conjunctivitis resolved and $N$ gonorrhoeae was not isolated from the conjunctivae in two babies (cases 4 and 7) who had been treated with penicillin. Although the initial specimens grew PPNG the isolates did respond to penicillin, probably because they were mixed colonies or weak penicillinase producers.

Five $(50 \%)$ of 10 babies showed partial improvement only, with either culture or smear or both giving a positive result on the third day. Three babies (cases 1,2 , and 5) had a persistent discharge and positive results to smears and cultures despite treatment with penicillin.

TABLE I Anogenital gonococcal infections and cases of ophthalmia neonatorum in Singapore between 1976 and 1980

\begin{tabular}{|c|c|c|c|c|c|}
\hline & \multicolumn{5}{|l|}{ Year } \\
\hline & 1976 & 1977 & 1978 & 1979 & 1980 \\
\hline $\begin{array}{l}\text { Total No of } \\
\text { cases of gonorrhoea }\end{array}$ & 4076 & 7668 & 9052 & 9332 & 8303 \\
\hline $\begin{array}{l}\text { Percentage due to } \\
\text { PPNG strains } \\
\text { Total No of cases }\end{array}$ & $0 \cdot 1$ & $0 \cdot 3$ & $11 \cdot 2$ & $19 \cdot 2$ & $29 \cdot 9$ \\
\hline $\begin{array}{l}\text { Total No of cases } \\
\text { of ophthalmia neonatorum } \\
\text { No }(\%) \text { of cases due }\end{array}$ & 44 & 24 & 22 & 24 & 23 \\
\hline to PPNG strains & 0 & 0 & $2(9 \cdot 1)$ & $2(8 \cdot 3)$ & $8(34 \cdot 8)$ \\
\hline
\end{tabular}

PPNG = penicillinase-producing $N$ gonorrhoeae

TABLE II Response to initial treatment in 11 cases of ophthalmia neonatorum due to PPNG

\begin{tabular}{llll}
\hline Case No & Antibiotic Eyedrops & Clinical response & Bacteriological result \\
\hline 1 & Penicillin & $\begin{array}{c}\text { Initial improvement and relapse of discharge } \\
\text { after two days } \\
\text { Persistent discharge }\end{array}$ & $\begin{array}{c}\text { Smear positive/culture positive } \\
\text { (3rd day) }\end{array}$ \\
2 & Chloramphenicol & Sartial improvement & Smear negative/culture positive \\
3 & Penicillin & Cleared completely & Smear negative/culture negative \\
4 & Penicillin & Persistent discharge & Smear positive/culture positive \\
5 & Penicillin & Partial improvement & Smear negative/culture positive \\
6 & Penicillin & Cleared completely & Smear negative/culture negative \\
7 & Penicillin & Partial response & Smear positive/culture negative \\
8 & Penicillin & Partial response & Smear positive/culture positive \\
9 & Penicillin & Partial response & Smear negative/culture positive \\
10 & Kanamycin & Cleared completely & Smear negative/culture negative \\
11 & & &
\end{tabular}

PPNG = penicillinase-producing $N$ gonorrhoeae 
Babies with ophthalmia neonatorum due to PPNG were treated with $1 \%$ kanamycin eyedrops and intramuscular injections of $0.5 \mathrm{~g}$ kanamycin.

By the third day of treatment all babies were symptomfree and had negative culture results.

\section{INVESTIGATION OF PARENTS}

All 11 mothers were investigated; five had no symptoms but three complained of a discharge which was not distinguishable from the lochia after delivery, and three gave a history of abnormal discharge between one month and one week before delivery.

$N$ gonorrhoeae was isolated from material from each of the parents; only five were infected with PPNG strains. This difference was noted in a previous study ${ }^{4}$ of prostitutes and their contacts. These women had not had sexual contact with their husbands for less than one month before delivery.

Only nine of the 11 fathers were available for investigation. None had symptoms, and gonococci were isolated from the urethra of only two of these men; both strains were non-PPNG. Five men had been treated previously for urethral discharge by private practitioners. Although two men gave no history of treatment the organism was not cultured from the urethra.

\section{Discussion}

This study highlights the need to consider PPNG infection as a cause of ophthalmia neonatorum, especially in areas with a high prevalence of PPNG strains, which include the whole of South-east Asia and certain parts of the Pacific basin. Although there was some delay in starting definitive treatment no permanent sequelae were noted at short-term follow up.

Ophthalmia neonatorum caused by penicillinsensitive strains of $N$ gonorrhoeae usually resolves within three days of the start of treatment. In ophthalmia neonatorum due to PPNG resolution by the third day had not occurred. Except for this fact, there was no clinical difference between PPNG and non-PPNG infections.

Non-PPNG strains were isolated in six of the 11 mothers. In a previous study from Singapore ${ }^{4}$ about one-third of source contacts of patients with PPNG infections were infected with non-PPNG strains. This may be because gonococci lose the plasmid coding for $\beta$-lactamase production. Alternatively, there may be infection with both PPNG and nonPPNG strains, with the PPNG strains predominating on culture or being preferentially selected for testing.

In this study we have shown that $1 \%$ kanamycin eyedrops used for three days together with kanamycin in a single intramuscular injection of $0.5 \mathrm{~g}$ is adequate treatment for ophthalmia neonatorum due to PPNG. We were obliged to give the parenteral treatment since we had previously had a poor response when eyedrops alone were used. ${ }^{4}$ Although there may be reservations on the use of kanamycin in young infants the dose used was within safe limits. An intramuscular injection of $0.5 \mathrm{~g}$ kanamycin leads to a peak concentration of above $20 \mu \mathrm{g} / \mathrm{ml}$ within two hours. Acute kanamycin toxicity most commonly occurs with concentrations higher than $30 \mu \mathrm{g} / \mathrm{ml}$. The neonate has not achieved its full normal renal function. Only one single dose of kanamycin, however, is given in the treatment of gonorrhoea. Most cases of ototoxicity occur when kanamycin is used for more than 14 days. In 1978 when we first encountered ophthalmia neonatorum due to PPNG there was no definitive or established treatment schedules. The antibiotics available were spectinomycin and kanamycin. As its toxicity in neonates is not known spectinomycin should be avoided in the treatment of ophthalmia neonatorum. Long-term follow up is needed to ascertain the ototoxic effects of the drug in these children. Although eyedrops are not available, the third generation cephalosporins may be a possible alternative.

Since the latter half of 1980 the antibiotic of choice in Singapore for uncomplicated gonorrhoea has been kanamycin with second-line choices being spectinomcyin and cefotaxime. Treatment schedules for complications such as epididymitis, pelvic inflammatory disease, and ophthalmia neonatorum have not been established. Spectinomycin, cefuroxime, and cefotaxime are expensive and, moreover, are given parenterally. Presently, the problem of PPNG is confined to certain areas of the world. With everincreasing travel, however, the problem could become worldwide. A pooling of resources and manpower is necessary to draw up suitable treatment schedules for complications caused by PPNG.

\section{References}

1. Dunlop EMC. Non-specific genital infection: laboratory aspects. In: Morton RS, Harris IRW, eds. Recent Advances in Sexually Transmitted Diseases. London: Churchill Livingstone, 1975.

2. Rajan VS, Sng EH, Khoo R, et al. Beta-lactamase strains of gonococci in Singapore. Asian J Infect Dis 1977; 1:62.

3. Pang R, Teh LB, Rajan VS, et al. Gonococcal ophthalmia neonatorum caused by beta-lactamase-producing Neisseria gonorrhoeae. $\mathrm{Br}$ Med $J$ 1979; i: 380.

4. Rajan VS, Thirumoorthy T, Tan NJ. Epidemiology of penicillinase-producing Neisseria gonorrhoeae in Singapore. $\mathrm{Br}$ $J$ Vener Dis 1981; 57: 158-61.

5. Rajan VS, Pang R, Sng EH. An evaluation of treatment in gonococcal ophthalmia neonatorum. Singapore Med J 1978; 19:86-8.

6. Bunn PA. Kanamycin. Med Clin North Am 1970;54: 1245-56. 